\title{
Review
}

\section{Role of Exercise and Dietary Supplementation in Attenuation of Traumatic Brain Injury in American Football}

\author{
Kyle Rank, BS; Giovanna Ramos, BS; Cameron Addie, BS, Tyler Neltner, BS; Mike Fraser, BS; Ludmila M. Cosio-Lima, PhD; \\ Christopher Wirth, PhD; Debra Vinci, DrPh; Youngil Lee, PhD*
}

Department of Movement Sciences and Health, University of West Florida, Pensacola, FL 325 I 4, USA

\section{"Corresponding author}

Youngil Lee, PhD

Molecular and Cellular Exercise Physiology Laboratory, Usha Kundu College of Health University of West Florida, I I000 University Pkwy, Bldg.72, Pensacola, Florida, 325 I4, USA; Tel. I-850-474-2596; Fax: I-850-474-2596; E-mail: ylee@uwf.edu

\section{Article information}

Received: December $4^{\text {th }}, 2018$; Revised: January $5^{\text {th }}, 2019$; Accepted: January $5^{\text {th }}$, 2019; Published: January $7^{\text {th }}, 2019$

\section{Cite this article}

Rank K, Ramos G,Addie C, et al. Role of exercise and dietary supplementation in attenuation of traumatic brain injury in american football. Sport Exerc Med Open J. 2019; 5(I): 5-I0. doi: 10.17140/SEMOJ-5-168

\section{ABSTRACT |}

Given the prevalence of traumatic brain injuries (TBI) in contact sports such as American Football, the need for increased research in TBI has been dramatically increased over the last 20-years. TBI has two main mechanisms that cause neuronal cell death following an incident: direct axonal death and neuronal inflammation, with the latter being the most common because it persists more than a decade and chronically affects neighboring neurons. Therefore, proper management that reduces inflammation post-TBI should be stressed in order to facilitate propitious recovery. While sideline concussion protocols have been implemented in sports fields, it is important immediately to initiate recovery protocols in order to minimize the degree of progressive neuronal death caused by TBI. While difficult to individualize symptoms for each occurrence, it is essential to incorporate a pretest of cognition, memory, and balance as a means of determining the severity of TBI. Although the sports concussion assessment tool 2 has been used by collegiate and professional teams, this tool is just based on observation and comparison. Thus, more precise and advanced diagnosis using biological methods are needed to accurately assess individual symptoms, which should save lives. One example is to use serum creatine kinase (CK) levels because CK released from damaged brain tissues enters the bloodstream and thus TBI can be quickly assessed and identified. While the immediate diagnosis of TBI is one part of management, efficacious treatments of post-TBI is also critical. Regarding this, exercise and nutritional supplementation have been reported to be effective. While specific pathways of neuroprotective mechanisms remain to be elucidated, endurance exercise along with supplementation of fish oil, caffeine, and vitamin D seems to elicit neuroprotective effects. This review provides potential mechanisms responsible for exercise and nutritional supplementation-mediated neuroprotection against TBI. Since human subjects are limited to mechanistic studies requiring invasive surgical procedures, research involving animals (e.g., mouse and rat) are also introduced in this review.

Keywords

Exercise; Traumatic brain injury; Concussion; Vitamin D; Caffeine, Omega-3 fatty acid; American football.

\section{INTRODUCTION}

A pproximately 1.5 million Americans experience a traumat- ic brain injury (TBI) each year. ${ }^{1,2}$ TBI's can occur in many ways such as car accidents, falls, work-related injuries, and athletic events, leaving lasting effects that may lead to degenerative diseas- es. ${ }^{3}$ There are approximately five million Americans that currently living with these TBI-related disabilities. ${ }^{2,4}$ TBI has traditionally been diagnosed in a clinical setting with the use of sophisticated medical techniques such as magnetic resonance imaging (MRI) or cognitive function tests. Numerous studies have been conducted to identifying the main causes of TBI and reported that oxida- 
tive stress and deficiencies in vitamin $\mathrm{D}$ and minerals are linked to TBI progression. Nevertheless, there has been no evidence of a comprehensive and successful treatment for TBI. ${ }^{5-9}$ Since many mechanisms (e.g., metabolic and cognitive decline) are implicated in TBI, potent interventions to help increase resiliency to a poor progression of TBI needs to be established. Indeed, although over 150 national football league players will be diagnosed with a concussion each year, ${ }^{10}$ there have not been many reliable resources that clearly illustrate recovery methods for TBI, while the emphasis in the industry at present focuses upon the protection of TBI by making the sport rules safer for players and the equipment more protective. Therefore, the purpose of this review was to provide an overview of current literature of TBI and introduce effective recovery strategies using exercise and supplementation to minimize neuro inflammation and cellular damage from a TBI event for American football players.

\section{MANAGEMENT OFTBI}

\section{Observational Diagnosis of TBI}

Sideline concussion protocols are being implemented in virtually every sport in the United States. As chronic traumatic encephalopathy (CTE) and the effects of TBI's have been brought to attention in the fields of contact sports, the need for quick diagnosis and management has become necessary. Professional and collegiate sports organization have begun to invest heavily into resources, ensuring the safety of athletes with potential TBI by implementing specific sideline and return to play protocols (e.g., SCAT2, SCAT3, and NFL modified) along with hiring experts in the field (e.g., physicians and athletic trainers).$^{10}$ While difficult to individualize symptoms for each event, sport teams have ensured that baseline testing is implemented before full participation in seasonal training to ensure that affected athlete's reach base levels of cognitive and physical function prior to returning to sport. ${ }^{11}$ In addition, upon returning to baseline, the athletes must pass a gradual exercise test to ensure TBI symptoms will not relapse, and the athlete can return to play without cumulating injury. ${ }^{11}$ In opposition to continuous organizational efforts for the prevention of poor progression of TBI, however, most athletes under report symptoms and injuries. For example, approximately $48 \%$ of high school players do not report symptomatic events of TBI. ${ }^{12}$ Therefore, educational promotion for improvement in student awareness about TBI (e.g., medical knowledge and consequence of TBI) should be frequently provided to athletes.

The athlete suffering from TBI is given information by a team physician to use outside of the team atmosphere in order to continue recovery. ${ }^{11}$ However, evidence has shown that the athletes do not seem to receive individualized information about timing, intensity, or type of treatment but to be informed about general guidelines. ${ }^{11}$ Therefore, there arises a need for more complete empirical evidence in diagnosis and management. ${ }^{12}$

\section{Use of Creatine Kinase as a TBI Biomarker}

Given that general field, tests cannot precisely diagnose nor iden- tify the levels of TBI, measurement of a biomarker is necessary for detection of TBI. The need for immediate and precise diagnosis has shifted the medical communities' focus from clinical observational methods to the use of biological methods, such as bloodborne markers. ${ }^{13}$ Regarding this, creatine kinase $(\mathrm{CK})$ has been shown to be a reliable metabolic marker of damaged tissues and can be measured by less invasive measures. ${ }^{14-16}$

Acute damage to tissue normally results in the release of extracellular enzymes into the blood. One such enzyme (marker) that is present after brain injuries are CK. Elevated levels of CK in the blood has long been used as a marker for tissue damage in tissues like the brain and heart. CK is normally not present in the blood; however, after the onset of an acute brain injury, it is present in the peripheral blood. ${ }^{17}$ Similarly, a recent study by Karkela et al demonstrated that a brain-specific creatine kinase (CK-BB) is also present in cerebrospinal fluid after a hypoxic brain injury. ${ }^{18}$ This group also found that increased levels of neuron-specific enolase (NSE), present in cerebrospinal fluid is present in the blood after a hypoxic brain injury.

\section{New Blood Biomarker for Chronic Traumatic Encephalopathy}

In the past, creatine kinase was considered as the only reliable technique for medical professionals to detect the possibility of a TBI. However, emerging research has shown that there is a more accurate marker of brain injury than CK. Cherry et al have recently discovered that a brain-specific protein, CLL11, is increased in the blood who have been diagnosed with CTE. ${ }^{19}$ Based on their hypothesis this could act as a new diagnostic tool that could improve diagnosis time from days to potentially hours. In a sport such as American football, this could arguably be a most positive change in protecting players brain health since the 1950s adoption of polymer, hard shell helmets.

\section{EXERCISE INTERVENTION ANDTBI RECOVERY}

Aerobic exercise has emerged as a potent intervention to prevent poor progression of various neurodegenerative diseases because of its key role in decreasing inflammation and modulating favorable gene expression. Neural inflammation is a secondary cause of neuronal cell death due to the excessive release of proinflammatory cytokines from immune cells and tissues of an injured area. ${ }^{20}$ Inflammation has been reported to be a prime source of both extrinsic (e.g., cytokine-mediated) and intrinsic (e.g., mitochondriamediated) cell death since it activates cell death executioner protein, caspase $3 .{ }^{21}$ A previous animal study has shown that endurance exercise (e.g., treadmill and wheel exercise) decreases active caspase 3 levels in the hippocampus following a TBI instance, ${ }^{20}$ resulting in attenuation of neuronal death and improved memory retention, and cognitive abilities. ${ }^{20}$ Given that neuronal cells are post-mitotic cells possessing an extremely low regenerative capacity, it is reasonable to speculate that improvement in neurogenesis may be a potential mechanism responsible for exercise-induced restoration of neuronal function. Indeed, supporting this supposition, studies using animals have demonstrated that treadmill running exercise elicits remarkable neural regeneration..$^{22-24}$ 
Given the high rate of brain trauma in football players, this exercise-induced neurogenesis mechanism will be an important therapeutic strategy; however, despite this therapeutic potential, it has been recommended that physical activities should be limited until symptoms disappear. ${ }^{20,25}$ For example, a study has shown that abstaining from exercise for five weeks following brain trauma significantly reduces neuroinflammation and cognitive dysfunction when compared to exercise intervention imposed one week after the incident. ${ }^{20,26}$ These studies also showed that the lesion size and inflammation in the brain were significantly suppressed upon the delayed onset of exercise therapy. Thus, current research suggests an important notion that while exercise can be a potent intervention for neuroprotection via neurogenesis, initiating exercise too soon without an appropriate recovery period from the brain trauma and high intensity may exacerbate the symptom.

\section{NUTRITIONAL SUPPLEMENTATION ANDTBI RECOVERY -}

\section{Creatine Supplementation and TBI}

Creatine is a nitrogenous organic compound that is found in vertebrates and most popular among athletics due to its potential role in muscular hypertrophy. The main role of creatine is the recycling of adenosine diphosphate back into adenosine triphosphate. It also plays a role in the moderation of oxidative stress ${ }^{27}$ and apoptotic effects in neurons, both of which are beneficial to brain health against TBI. ${ }^{28,29}$

A clinical trial examining the effect of creatine supplementation on mild TBIs has reported that $0.4 \mathrm{~g} / \mathrm{kg}$ of creatine given to children and adolescents suffering from TBI improved several physiological symptoms including post-traumatic amnesia, communication, and cognitive function. ${ }^{30}$ Although encouraging, these results are limited due to many unexplained factors such as the regenerative ability of an adolescent brain and potential factors pertaining to the cause of TBIs.

\section{Omega-3 Fatty Acids and TBI}

Fish oils are a supplement high in omega-3 fatty acids and have shown to be efficacious in reversing the detrimental metabolic symptoms of TBI leading to a cognitive decline in many studies. ${ }^{6,7,931}$ Omega-3 fatty acids are polyunsaturated fatty acids and abundantly found in the brain. They are very important components of the plasma membrane and associated with the neuronal signaling of the brain. ${ }^{32}$

Docosahexaenoic acid, one of the omega-3 fatty acids, has been reported to enhance expression of brain-derived neurotrophic factor and to be necessary to maintain the molecular processes required for a proper cognitive function, neuronal excitability, learning process, memory retention, and gene expression. ${ }^{9,33}$ In this regard, previous studies have shown a positive outcome from a diet rich in omega-3 fatty acid against TBI. For example, studies led by Wu et al have reported that football players, who have suffered TBI's improved learning process, brain plasticity, and cognitive function., ${ }^{9,31}$
Silent information regulator 2 (Sir2) is an important protein for sustaining cellular homeostasis in the brain..$^{31,34,35}$ Interestingly, it has been reported that Sir2 diminishes in the brain suffering TBI, ${ }^{35}$ whereas a rich diet on omega 3 fatty acids helps to restore the levels of Sir2, resulting in reducing cognitive dysfunction and increasing plasticity of the brain..$^{9,31}$

\section{Caffeine Supplementation and TBI}

Caffeine is a psychomotor stimulant in the brain region of the basal ganglia. ${ }^{36}$ The molecular mechanism of caffeine is the activation of adrenergic tone via inhibiting adenosine A1 receptors, which oppose beta-adrenergic signaling cascades by inhibiting an adenylate cyclase. ${ }^{36-38}$ Supporting the findings above, recent studies have demonstrated that psychomotor stimulation via the caffeineinduced A1 receptor blockage protects the brain against both acute and chronic TBI. ${ }^{38,39}$ In addition, caffeine-mediated adenosine A2a receptor inhibition has been reported to facilitate neuroprotection by increasing neurotransmitter releases and stimulating neurosensory activities. ${ }^{36,40,41}$ Indeed, studies using both human and animals showed that caffeine supplementation after TBI provide beneficial effect against TBI. ${ }^{38,42}$

Parkinson's disease (PD) is caused by degeneration of dopaminergic nigrostriatal neurons of the basal ganglia, characterized by a series of motor disturbances. ${ }^{37}$ Although advanced age and genetic mutation are well-accepted etiology of PD, it can also arise from severe TBIs frequently induced by contact or noncontact sports, car crashes or anything else where a possible traumatic impact can occur in the head region. ${ }^{43-45}$ Regarding this, recent studies has demonstrated that caffeine administration increases extracellular dopamine in the striatum in the striatum. ${ }^{46,47}$ This indicates that caffeine supplementation may be a potential therapeutic strategy; however, given that maintenance of basal activation of adenosine A1 and A2a receptors plays an important role in antiinflammation, vasodilation, pain modulation, anti-arrhythmia, and metabolic regulation, it is important to understand that the chronic blockage via mega dose ( $>400 \mathrm{mg}$ per day for adults) of caffeine or pharmacological antagonists against thereceptors may cause several adverse effects.

\section{Vitamin D Supplementation}

Vitamin D is a lipid-soluble essential vitamin and has been known to play a critical role in maintaining calcium balance. Importantly, vitamin $\mathrm{D}$ is also involved in the molecular processes of cell proliferation, differentiation, and survival since it can function as a hormone ligand that binds to the nuclear receptor vitamin D receptor, resulting in the transcription of vitamin D-responsive genes. ${ }^{48}$ Vitamin D can be made by de novo synthesis from cholesterol through exposure of skin to ultraviolet $\mathrm{B}$ and taken by dietary sources (plants and animals) or supplements. Vitamin D2 and D3 derived from plants and animals, respectively should go through the hydroxylation processes via first the liver and kidney, resulting in the active hormonal form of vitamin $\mathrm{D}(1,25$-dihydroxy vitamin $\mathrm{D}$ ) or calcitriol of note, previous studies have shown that vitamin D3 has a more efficacious in raising an active form of 
vitamin D. ${ }^{49}$

Vitamin D deficiency is associated with impaired cognitive function and has been shown to be a common symptom in patients suffering from a TBI. ${ }^{50} \mathrm{~A}$ study found that patients suffering from TBI with a vitamin D deficiency had significantly lower score on a cognitive examination test than those who did not $(p=0.034) .{ }^{50}$ On the contrary, in an animal study, supplementation of calcitriol, an active vitamin D metabolite, significantly decreases cell death after TBI in the cortex region of the rat and improves cognitive function. ${ }^{51}$ This study supports the notion that vitamin D supplementation helps sustain higher levels of cognitive function after a TBI. Furthermore, growing evidence has revealed that the administration of progesterone in combination with vitamin D significantly improves cognitive recovery after a TBI. ${ }^{52,53}$ These studies indicate that vitamin D supplementation may help to offset some of the symptoms and improve recovery from a TBI.

\section{SUMMARY}

The high number of concussions in sports with high contact level such as football, generated a great interest to search penitential interventions to maintain brain health throughout athletes' carrier and avoid TBI that results in critical health problems. Exact molecular pathways of TBI progression still remains unclear; however, it is apparent that cellular metabolism has a significant impact on the regulation of TBI recovery. While recovery from cognitive impairment can occur within 3-7-days post-TBI, cognitive deterioration has been shown to continue during the recovery period. Current clinical protocols mandate a limitation of physical activities until symptoms reside, with cognitive function returning to normal levels since immoderate physical exertion exacerbates TBI symptoms. Accumulating evidence suggests that aerobic exercise with moderate intensity and nutritional supplementation may improve recovery from TBI and reduced poor progression of TBI. However, their neuroprotective mechanisms remain to be elucidated neurogenesis in response to moderate exercise and supplementation (e.g., fish oil, vitamin D, and caffeine) play a key role in expediting faster recovery period. More importantly, the combination of moderate exercise with nutritional supplementation may complement the national football league (NFL) or national collegiate athletic association (NCAA) return to play protocol. However, since the degree of symptoms from TBI can be drastically different based on severity and the individual, it is beyond the scope of this review to recommend specific dosages of supplementation. Thus, future studies are necessary to explore the current limitations in the future.

\section{ACKNOWLEDGEMENT}

This project was supported by a research grant from the University of West Florida through office of research and sponsored srograms (R0062) and UWF Florida Research Fellowship to YL (CF6672 and CR0070).

\section{CONFLICTS OF INTEREST}

The authors declare that they have no conflicts of interest.

\section{REFERENCES}

1. Faul MXL, Wald MM, Coronado V, Dellinger AM. Traumatic brain injury in the United States: National estimates of prevalence and incidence, 2002-2006. Injury Prevention. 2010; 16(1): 1. doi: 10.1136/ip.2010.029215.951

2. Thurman DJ, Alverson C, Dunn KA, Guerrero J, Sniezek JE. Traumatic brain injury in the United States: A public health perspective. J Head Trauma Rehabil. 1999;14(6): 602-615.

3. Corrigan JD, Hammond FM. Traumatic brain injury as a chronic health condition. Arch Phys Med Rehabil. 2013; 94(6): 1199-1201. doi: 10.1016/j.apmr.2013.01.023

4. Gardner RC, Langa KM, Yaffe K. Subjective and objective cognitive function among older adults with a history of traumatic brain injury: A population-based cohort study. PLoS Med. 2017; 14(3): e1002246. doi: 10.1371/journal.pmed.1002246

5. Cope EC, Morris DR, Scrimgeour AG, VanLandingham JW, Levenson CW. Zinc supplementation provides behavioral resiliency in a rat model of traumatic brain injury. Physiol Behav. 2011; 104(5): 942-947. doi: 10.1016/j.physbeh.2011.06.007

6. Mills JD, Bailes JE, Sedney CL, Hutchins H, Sears B. Omega-3 fatty acid supplementation and reduction of traumatic axonal injury in a rodent head injury model. J Neurosurg. 2011; 114(1): 77-84. doi: 10.3171/2010.5.JNS08914

7. Mills JD, Hadley K, Bailes JE. Dietary supplementation with the omega-3 fatty acid docosahexaenoic acid in traumatic brain injury. Neurosurgery. 2011; 68(2): 474-481. doi: 10.1227/ NEU.0b013e3181ff692b

8. Moro N, Katayama Y, Igarashi T, Mori T, Kawamata T, Kojima J. Hyponatremia in patients with traumatic brain injury: Incidence, mechanism, and response to sodium supplementation or retention therapy with hydrocortisone. Surg Neurol. 2007; 68(4): 387-393. doi: 10.1016/j.surneu.2006.11.052

9. Wu A, Ying Z, Gomez-Pinilla F. Dietary omega-3 fatty acids normalize BDNF levels, reduce oxidative damage, and counteract learning disability after traumatic brain injury in rats. J Neurotrauma. 2004; 21(10): 1457-1467. doi: 10.1089/neu.2004.21.1457

10. National Football League (NFL). Diagnosis and Management of Concussion. 2016.

11. NCAA. Concussion Diagnosis and Management Best Practices. NCAA Sports Science. 2016.

12. Putukian M. The acute symptoms of sport-related concussion: diagnosis and on-field management. Clin Sports Med. 2011; 30(1): 49-61. doi: 10.1016/j.csm.2010.09.005

13. Schmid KE, Tortella FC. The diagnosis of traumatic brain injury on the battlefield. Front Neurol. 2012; 3: 90. doi: 10.3389\%2Ff- 
neur.2012.00090

14. Toman E, Harrisson S, Belli T. Biomarkers in traumatic brain injury: A review. J R Army Med Corps. 2016; 162(2): 103-108. doi: 10.1136/jramc-2015-000517

15. Sharma R, Rosenberg A, Bennett ER, Laskowitz DT, Acheson SK. A blood-based biomarker panel to risk-stratify mild traumatic brain injury. PloS One. 2017; 12(3): e0173798. doi: 10.1371/journal. pone. 0173798

16. Boeck CR, Carbonera LS, Milioli ME, et al. Mitochondrial respiratory chain and creatine kinase activities following trauma brain injury in brain of mice preconditioned with N-methyl-Daspartate. Mol Cell Biochem. 2013; 384(1-2): 129-137. doi: 10.1007/ s11010-013-1790-8

17. Somer H, Kaste M, Troupp H, Konttinen A. Brain creatine kinase in blood after acute brain injury. J Neurol Neurosurg Psychiatry. 1975 Jun; 38(6): 572-576.

18. Karkela J, Bock E, Kaukinen S. CSF and serum brain-specific creatine kinase isoenzyme (CK-BB), neuron-specific enolase (NSE) and neural cell adhesion molecule (NCAM) as prognostic markers for hypoxic brain injury after cardiac arrest in man. J Neurol Sci. 1993; 116(1): 100-109.

19. Cherry JD, Stein TD, Tripodis Y, et al. CCL11 is increased in the CNS in chronic traumatic encephalopathy but not in Alzheimer's disease. PLoS One. 2017; 12(9): e0185541. doi: 10.1371/journal.pone. 0185541

20. Piao CS, Stoica BA, Wu J, et al. Late exercise reduces neuroinflammation and cognitive dysfunction after traumatic brain injury. Neurobiol Dis. 2013; 54: 252-63. doi: 10.1016/j.nbd.2012.12.017

21. Glushakov AO, Glushakova OY, Korol TY, et al. Chronic upregulation of cleaved-caspase-3 associated with chronic myelin pathology and microvascular reorganization in the thalamus after traumatic brain injury in rats. Int J Mol Sci. 2018; 19(10). doi: 10.3390/ijms19103151

22. Itoh T, Imano M, Nishida S, et al. Exercise increases neural stem cell proliferation surrounding the area of damage following rat traumatic brain injury. I Neural Transm (Vienna). 2011; 118(2): 193-202. doi: 10.1007/s00702-010-0495-3

23. Kim H, Heo HI, Kim DH, et al. Treadmill exercise and methylphenidate ameliorate symptoms of attention deficit/hyperactivity disorder through enhancing dopamine synthesis and brain-derived neurotrophic factor expression in spontaneous hypertensive rats. Neurosci Lett. 2011; 504(1): 35-39. doi: 10.1016/j.neulet.2011.08.052

24. Griesbach GS, Gomez-Pinilla F, Hovda DA. Time window for voluntary exercise-induced increases in hippocampal neuroplasticity molecules after traumatic brain injury is severity dependent. $J$ Neurotrauma. 2007; 24(7): 1161-7111. doi: 10.1089/neu.2006.0255
25. Weightman MM, Bolgla R, McCulloch KL, Peterson MD. Physical therapy recommendations for service members with mild traumatic brain injury. J Head Trauma Rehabil. 2010; 25(3): 206-218. doi: 10.1097/HTR.0b013e3181dc82d3

26. Yoon KJ, Kim DY. Immediate Effects of a single exercise on behavior and memory in the early period of traumatic brain injury in rats. Ann Rehabil Med. 2018; 42(5): 643-651. doi: 10.5535/ arm.2018.42.5.643. doi: 10.5535/arm.2018.42.5.643

27. Saraiva AL, Ferreira AP, Silva LF, et al. Creatine reduces oxidative stress markers but does not protect against seizure susceptibility after severe traumatic brain injury. Brain Res Bull. 2012; 87(2-3): 180-186. doi: 10.1016/j.brainresbull.2011.10.010

28. Ainsley Dean PJ, Arikan G, Opitz B, Sterr A. Potential for use of creatine supplementation following mild traumatic brain injury. Concussion. 2017; 2(2): Cnc34. doi: 10.2217/cnc-2016-0016

29. Freire Royes LF, Cassol G. The effects of creatine supplementation and physical exercise on traumatic brain injury. Mini Rev Med Chem. 2016; 16(1): 29-39.

30. Sakellaris G, Kotsiou M, Tamiolaki M, et al. Prevention of complications related to traumatic brain injury in children and adolescents with creatine administration: An open label randomized pilot study. J Trauma. 2006; 61(2): 322-329. doi: 10.1097/01. ta.0000230269.46108.d5

31. Wu A, Ying Z, Gomez-Pinilla F. Oxidative stress modulates Sir2alpha in rat hippocampus and cerebral cortex. Eur J Neurosci. 2006; 23(10): 2573-2580. doi: 10.1111/j.1460-9568.2006.04807.x

32. Wang T, Van KC, Gavitt BJ, et al. Effect of fish oil supplementation in a rat model of multiple mild traumatic brain injuries. Restor Neurol Neurosci. 2013; 31(5): 647-659. doi: 10.3233/RNN130316

33. Sun GY, Simonyi A, Fritsche KL, et al. Docosahexaenoic acid (DHA): An essential nutrient and a nutraceutical for brain health and diseases. Prostaglandins Leukot Essent Fatty Acids. 2018; 136: 3-13. doi: 10.1016/j.plefa.2017.03.006

34. Suzuki K, Koike T. Mammalian Sir2-related protein (SIRT) 2-mediated modulation of resistance to axonal degeneration in slow wallerian degeneration mice: A crucial role of tubulin deacetylation. Neuroscience. 2007; 147(3): 599-612.doi: 10.1016/j.neuroscience.2007.04.059

35. Wu A, Ying Z, Gomez-Pinilla F. Omega-3 fatty acids supplementation restores mechanisms that maintain brain homeostasis in traumatic brain injury. J Neurotrauma. 2007; 24(10): 1587-1595. doi: $10.1089 /$ neu.2007.0313

36. Fisone G, Borgkvist A, Usiello A. Caffeine as a psychomotor stimulant: mechanism of action. Cell Mol Life Sci. 2004; 61(7-8): 857-872. doi: 10.1007/s00018-003-3269-3 
37. Cuenca L, Gil-Martinez AL, Cano-Fernandez L, et al. Parkinson's disease: A short story of 200-years. Histol Histopathol. 2018; 18073. doi: 10.14670/HH-18-073

38. Lusardi TA, Lytle NK, Szybala C, Boison D. Caffeine prevents acute mortality after TBI in rats without increased morbidity. Exp Neurol. 2012; 234(1): 161-168. doi: 10.1016/j.expneurol.2011

39. Ning YL, Yang N, Chen X, et al. Chronic caffeine exposure attenuates blast-induced memory deficit in mice. Chin J Traumatol. 2015; 18(4): 204-211.

40. Kuzmin A, Johansson B, Gimenez L, Ogren SO, Fredholm $\mathrm{BB}$. Combination of adenosine $\mathrm{A} 1$ and $\mathrm{A} 2 \mathrm{~A}$ receptor blocking agents induces caffeine-like locomotor stimulation in mice. Eur Neuropsychopharmacol. 2006; 16(2): 129-136. doi: 10.1016/j.euroneuro.2005.07.001

41. Astorino TA, Rohmann RL, Firth K. Effect of caffeine ingestion on one-repetition maximum muscular strength. Eur J Appl Physiol. 2008; 102(2): 127-132. doi: 10.1007/s00421-007-0557-x

42. Sachse KT, Jackson EK, Wisniewski SR, et al. Increases in cerebrospinal fluid caffeine concentration are associated with favorable outcome after severe traumatic brain injury in humans. J Cereb Blood Flow Metab. 2008; 28(2): 395-401. doi: 10.1038/sj.jcbfm.9600539

43. Adams JW, Alvarez VE, Mez J, et al. Lewy body pathology and chronic traumatic encephalopathy associated with contact sports. J Neuropathol Exp Neurol. 2018; 77(9): 757-768. doi: 10.1093/jnen/ nly065

44. Pearce N, Gallo V, McElvenny D. Head trauma in sport and neurodegenerative disease: An issue whose time has come? Neurobiol Aging. 2015; 36(3): 1383-1389. doi: 10.1016/j.neurobiolaging.2014.12.024

45. Young JS, Hobbs JG, Bailes JE. The impact of traumatic brain injury on the aging brain. Curr Psychiatry Rep. 2016; 18(9): 81. doi: 10.1007/s11920-016-0719-9

46. Owolabi JO, Olatunji SY, Olanrewaju AJ. Caffeine and cannabis effects on vital neurotransmitters and enzymes in the brain tissue of juvenile experimental rats. Ann Neurosci. 2017; 24(2): 65-73. doi: $10.1159 \% 2 \mathrm{~F} 000475895$

47. Essawy SS, Tawfik MK, Korayem HE. Effects of adenosine receptor antagonists in MPTP mouse model of parkinson's disease: Mitochondrial DNA integrity. Arch Med Sci. 2017; 13(3): 659-669. doi: 10.5114/aoms.2017.67284

48. Fleet JC. What have genomic and proteomic approaches told us about vitamin D and cancer? Nutrition Reviews. 2007; 65(8 Pt 2): S127-S130.

49. Tripkovic L, Lambert H, Hart K, et al. Comparison of vitamin D2 and vitamin D3 supplementation in raising serum 25-hydroxyvitamin D status: A systematic review and meta-analysis. Am J Clin Nutr. 2012; 95(6): 1357-1364. doi: 10.3945/ajcn.111.031070

50. Jamall OA, Feeney C, Zaw-Linn J, et al. Prevalence and correlates of vitamin $\mathrm{D}$ deficiency in adults after traumatic brain injury. Clin Endocrinol (Oxf). 2016; 85(4): 636-644. doi: 10.1111/cen.13045

51. Cui C, Cui J, Jin F, et al. Induction of the vitamin D receptor attenuates autophagy dysfunction-mediated cell death following traumatic brain injury. Cell Physiol Biochem. 2017; 42(5): 1888-1896. doi: $10.1159 / 000479571$

52. Hua F, Reiss JI, Tang H, et al. Progesterone and low-dose vitamin D hormone treatment enhances sparing of memory following traumatic brain injury. Horm Behav. 2012; 61(4): 642-651.

53. Stein DG, Cekic MM. Progesterone and vitamin d hormone as a biologic treatment of traumatic brain injury in the aged. PM R. 2011; 3(6 Suppl 1): S100-S110. doi: 10.1016/j.pmrj.2011.03.010 